\title{
Surgery for bronchiectasis
}

\author{
Thirugnanam Agasthian
}

Mount Elizabeth Medical Centre, Singapore

Correspondence to: Dr. Thirugnanam Agasthian. Senior Consultant Thoracic Surgeon, 3 Mount Elizabeth, \#14-12, Mount Elizabeth Medical Centre, Singapore 228510. Email: t.agasthian@gmail.com.

\begin{abstract}
Current goals of surgery for bronchiectasis are to cure, improve quality of life and prevent life threatening complications after failed medical therapy. Best surgical outcomes are obtained after complete resections for localised disease. Symptom improvement can be obtained by surgery in selected diffuse disease. The article reviews the role and types of surgical resections for bronchiectasis.
\end{abstract}

Keywords: Surgery; pulmonary infections; bronchiectasis

Received: 26 July 2018; Accepted: 11 October 2018; Published: 12 November 2018.

doi: 10.21037 /jovs.2018.10.11

View this article at: http://dx.doi.org/10.21037/jovs.2018.10.11

\section{Introduction}

Though the incidence of bronchiectasis is declining worldwide it is still prevalent in developing countries due to post-bacterial and viral infections. In developed countries congenital causes are more common like immune deficiency syndromes (hypogammaglobulinemia, leukocyte dysfunction), metabolic defects (cystic fibrosis), ultrastructural defects (primary ciliary dyskinesia like Young and Kartegener's syndromes) and pulmonary sequestrations. With the re-emergence of tuberculosis worldwide the incidence of post tuberculous bronchiectasis has also increased to $11 \%$ of all TB patients $(1,2)$.

\section{Pathology}

Bronchiectasis is an irreversible dilatation of the peripheral bronchial airways secondary to damage of the bronchial wall. According to Cole's "vicious cycle" hypothesis, bronchiectasis develops on a background of genetic susceptibility and impaired mucosal clearance. Following a lung infection, there is persistence of virulent microorganisms in the bronchial tree, giving rise to a vicious cycle of repeated episodes of chronic inflammation with progressive airway and lung damage. The subsequent secondary enlargement of lymphoid follicles and inflammation in the bronchial wall of small airways gives rise to release of inflammatory enzymes causing damage to larger airways resulting in bronchial dilatation, increase in submucosal glands, loss of ciliary function and neovascularisation. The inflammation eventually spreads to lung parenchyma causing fibrosis. Although the larger airways get dilated in bronchiectasis, it is the inflammation of the small and medium airways that gives rise to airflow obstruction which often gives rise to a mixed obstructive, restrictive lung function pattern (2).

Bronchiectasis can be classified anatomically, functionally or by distribution patterns. Anatomically it can be classified on morphologic patterns into tubular characterised by cylindrical smooth dilatation of the bronchi, varicose with multiple tortuous dilatations of the airway and cystic with saccular dilatations of the bronchi terminating in blind ending sacs. Tubular is the mildest and cystic is the most severe form of bronchiectasis (Figure 1).

Functionally bronchiectasis can be classified into perfused and non-perfused types based on V/Q scans. Perfused type is usually cylindrical bronchiectasis with intact pulmonary artery flow and functioning lung while the non-perfused type involves predominantly cystic with an absent pulmonary artery flow with retrograde filling of the pulmonary artery from the systemic circulation with non-functioning lung. Based on morphological and functional classifications, tubular/cylindrical bronchiectasis has better prognosis than cystic varieties as it is an early form of bronchiectasis, reversible by medical therapy. Cystic bronchiectasis tends to be completely-destroyed lung with non-functioning and non-perfused lung. They tend 

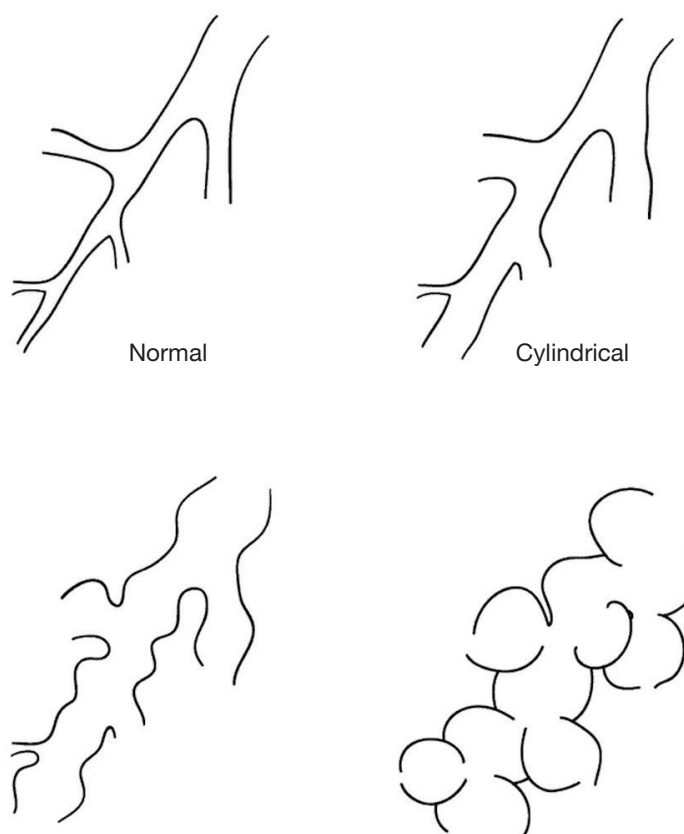

Varicose

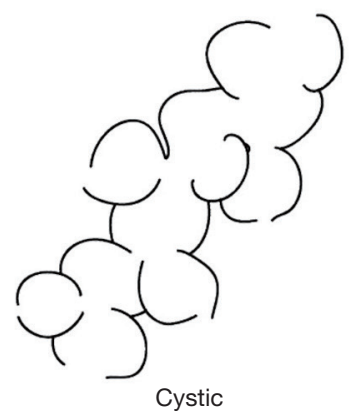

Figure 1 Classification of bronchiectasis by morphology.

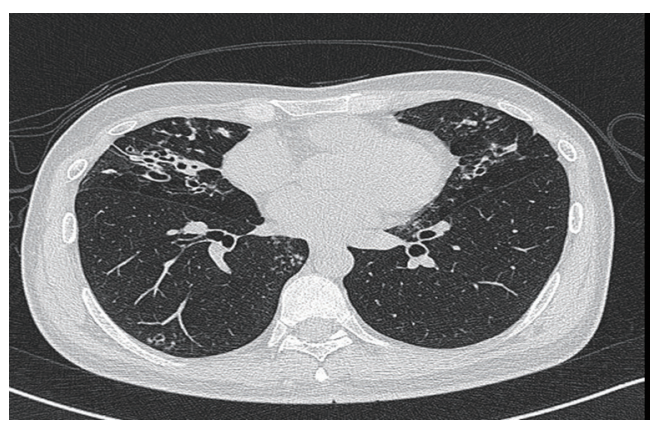

Figure 2 Middle lobe and lingular segment bronchiectasis.

to harbour the greatest virulent bacterial load especially Pseudomonas aeruginosa giving rise to severe symptoms and frequent crossover contamination of the healthier lung by infected sputum (2-5).

Bases on distribution patterns bronchiectasis can be localised (confined to a lobe or segments) or diffuse. The rate of localised involvement has been reported to be 3.6\% to $19 \%$. The left lung tends to be more involved than the right in $55 \%$ to $80 \%$ of cases due to the left main bronchi being narrower, longer and more acute. The lower lobe is the commonest lobe involved (30\%) followed by the middle lobe and lingular segments. The lower lobe basilar segments are commonly affected due to gravity dependent retention of infected secretions. The upper lobes are affected commonly in post tuberculous bronchiectasis and allergic bronchopulmonary aspergillosis (ABPA). Central airway bronchiectasis has been seen in cystic fibrosis, ABPA and Munier Kuhn syndrome. Right middle lobe and lingular segment bronchiectasis can be associated with non-tuberculous mycobacterium (NTM) infections (Lady Windermere syndrome, Figure 2). Enlarged tuberculous lymph nodes around the middle lobe bronchus may cause obstruction and secondary bronchiectasis. (Middle lobe syndrome) $(2,6,7)$.

Hemophilus influenza is the commonest pathogen isolated in bronchiectasis (29-70\%) followed by Pseudomonas aeruginosa (12-31\%). Despite being purulent 30\% to $40 \%$ of sputum samples failed to grow any pathogenic bacteria even with bronchoscopic bronchoalveolar lavage (BAL). P aeruginosa is associated with more extensive bronchiectasis, frequent exacerbations and accelerated decline in lung function. It also has the capacity to form biofilms which are impenetrable matrix around the bacterial wall resistant to the immune system and antibiotics. There is also a change in microbial flora with increasing severity of disease. Subjects with best preserved lung function have little or no isolated pathogens on sputum. As lung function declines $H$. influenza predominates with later colonisation by $P$. aeruginosa in progressive severe disease. Less common organisms in bronchiectasis are Klebsiella pneumoniae, Streptococcus pneumoniae and rarely Staph aureus. NTM are associated in $2 \%$ to $10 \%$ of bronchiectasis patients. It can be a primary cause of bronchiectasis (Lady Windermere syndrome) or a secondary opportunistic infection and colonisation where they can accelerate the rate of progression of bronchiectasis if left untreated (2).

\section{Indications for surgery}

The clinical course of non-cystic fibrosis bronchiectasis is variable as some have few symptoms and others exhibit progressive symptoms with loss of lung function over time (FEV1 loss at $10 \mathrm{~mL} /$ year). Factors associated with loss of accelerated lung function include cystic bronchiectasis, colonisation by Pseudomonas aeruginosa and repeated severe exacerbations. Chronic antibiotic treatment is currently not recommended except macrolide therapy which has antiinflammatory effects. Patients treated with long-term lowdose azithromycin showed decrease in exacerbations and sputum volume (2). Indications for surgery are based on two factors, failed medical therapy and extend of disease 
(localised $v s$. diffuse) $(2,8,9)$.

Failed medical therapy is inability to control symptoms with antibiotic therapy which are:

* Chronic daily cough with copious fetid sputum;

* Frequent infective exacerbations;

* Complications (hemoptysis, lung abscess and empyema);

* Prevention of possible progression of disease secondary to spillage contamination to healthy lung especially in cystic bronchiectasis;

* Colonisation by NTM that is resistant to prolonged medical therapy.

Indications by extend and distribution of disease can be classified into $(2,8)$ :

* Homogenous bronchiectasis: not surgical candidates because of diffuse predominantly cystic or saccular bronchiectasis affecting most parts of the lung with no good intervening functional lung function on $\mathrm{V} /$ Q scan. Most of these patients are treated medically and will progress to respiratory failure requiring bilateral single lung transplantations;

* Heterogenous bronchiectasis: surgical candidates depending on the type of heterogenous bronchiectasis which can be further subdivided into:

- Unilateral localised bronchiectasis: disease confined to a segment, lobe or completelydestroyed lung. They have the best outcomes with surgery as the single most important prognostic factor for good surgical outcomes is complete resection of all diseased lung;

- Bilateral localised bronchiectasis: multi-segmental/ lobar unilateral or bilateral disease with good intervening normal or minimally diseased lung. This pattern commonly seen in non-tuberculous infections. Complete surgical resection should be attempted if lung function permits Resections include combinations of lobectomy with segmentectomy or wedge resections. Surgery can be staged or done on same siting;

- Bilateral diffuse bronchiectasis: Bilateral diffuse bronchiectasis with localised cystic non-functioning, non-perfused lung on background of tubular bronchiectasis with perfused functioning lung. Though surgical cure rates are lower in this group (30\%) in selected cases, limited resection of most diseased cystic bronchiectatic lungs has had significant improvement of symptoms, less exacerbations, reduction in hospital stay, reduced antibiotic usage with overall improved quality of life. There is delay or halt in progression of disease due to early prevention of spillage contamination of adjacent healthier lung with virulent bacteria. The extent of symptom improvement will depend on the severity of residual background bronchiectasis. Best outcomes are seen in localised cystic bronchiectasis on a background of traction bronchiectasis which are non-progressive with low grade symptoms well controlled with medical therapy.

\section{Pre-operative surgical management}

Pre-operative bronchoscopy is indicated in localised bronchiectasis in a single lobe where a foreign body, stricture or tumour is suspected. Bronchoscopy with BAL and protected sputum collection is indicated if NTM infection is suspected. Identification of surgically targetable areas for successful surgical outcomes can be planned with pre-operative high-resolution CT scans, V/Q scan and lung function studies.

Acute, active infections and exacerbations should be treated aggressively before surgery with antibiotics and chest physiotherapy with postural drainage. Surgery should be done electively when the infection is adequately controlled as this decreases risks of post-operative empyema and bronchopleural fistula (BPF). Mucoid sputum has less elastase than purulent sputum and is less injurious to the lung and airways. Although complete bacterial eradication is not possible before surgery antibiotic therapy should be continued till sputum volume is minimal and nonpurulent. Any large abscess cavities should be drained preoperatively to prevent intraoperative spillage. Patients with severe bronchiectasis are admitted $48 \mathrm{~h}$ before surgery for intensive chest physiotherapy, postural drainage with vibratory massage. This is continued into the post-operative period. Prophylactic antibiotics are given at anaesthetic induction and continued for $48 \mathrm{~h}$ post operatively (2).

\section{Anaesthetic management}

Anaesthetic management is very crucial for safe conduct of surgery for bronchiectasis. Frequent suctioning of airway secretions and accurate lung isolation under bronchoscopic guidance is mandatory before commencement of surgery to 
prevent spillage and contamination of infected secretions to contralateral lung during surgical manipulation. As surgery for bronchiectasis is associated with dense vascular adhesions, good lung isolation gives good exposure to all parts of the chest for safe dissection under direct visualisation.

In bilateral bronchiectasis, to prevent accumulation of secretions in the non-operative lung which can cause troublesome intraoperative oxygen desaturation, frequent bronchial toilet by the anaesthesiologist is important. A left sided double lumen endotracheal tube is routine unless a left pneumonectomy is performed. In children and young adults in whom a double lumen endotracheal tube is too big, a bronchial blocker can be used.

In bilateral resections, whether staged or same siting, anaesthetic considerations are important. Selecting which side to operate first will be based on pre-operative $\mathrm{V} / \mathrm{Q}$ scan, differential lung function tests and extent of resection. For bilateral synchronous resections the better functioning side should be operated first to allow for safe single lung isolation when operating on the more diseased lung. In some cases where a previous lobectomy has been done contralateral side, patient may not be able to tolerate single lung ventilation and may need ancillary measures such as continuous positive airway pressure CPAP) at $5-10 \mathrm{mmHg}$ pressure or selective lobar blocker can be applied on the operative lung $(9,10)$ (Figure 3).

\section{Surgical technique}

\section{Open vs. minimally invasive surgery (MIS)}

Posterolateral thoracotomy continues to be the standard open approach of choice for bronchiectasis. It is well tolerated and offers good exposure to all parts of the chest and allows for tackling any major intraoperative complications. Whenever possible a muscle sparing thoracotomy should be done to minimise shoulder and chest-wall dysfunction as well as possible use of the latissimus dorsi or serratus anterior muscles in the event of post-operative BPF. Bilateral resections through thoracotomy needs to be staged because of the greater pain and impairment of lung function involved. Staged bilateral resections are done after full recovery usually after an interval of 4 to 6 weeks (12-15).

For synchronous bilateral resections, bilateral anterior thoracotomy or median sternotomy can be used. Both incisions give adequate exposure for bilateral resections like

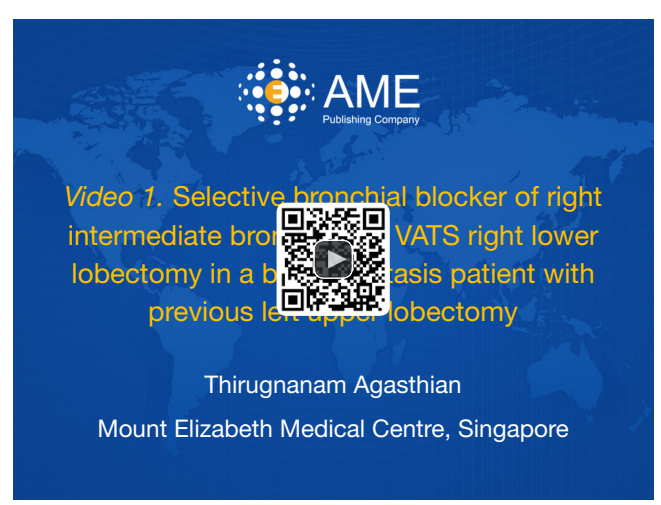

Figure 3 Selective bronchial blocker of right intermediate bronchus for VATS right lower lobectomy in a bronchiectasis patient with previous left upper lobectomy (11). VATS, videoassisted thoracoscopic surgery.

Available online: http://www.asvide.com/article/view/28191

the middle lobe and lingular segments (Figure 2). Median sternotomy gives good exposure to all lobes except the left lower lobe which may then need a left hemi-clam shell anterior thoracotomy extension to the sternotomy. It is safer approach for completion pneumonectomy or extrapleural or intrapericardial pneumonectomy for completely fused pleura with dense fibrotic hilum from inflammation. It allows early safe intra-pericardial access and ligation of the major hilar structures and prevention of contamination of the post pneumonectomy space. Bilateral anterior thoracotomy gives better exposure to all parts of the chest as compared to sternotomy especially to the posterior and diaphragmatic adhesions but causes more pain and chestwall trauma with impairment of lung function (2).

With increasing experience of MIS (video-assisted thoracoscopic surgery or robotic-assisted thoracic surgery) for lung cancer, it is increasingly used for inflammatory conditions. Its surgical advantage over open surgery is its superior exposure and visualisation to all parts of the chest especially the apex and the diaphragm due to superior optics, magnification and lighting. It causes lesser pain, chest-wall trauma and derangement of immunity with better preservation of post-operative lung function. It is very beneficial in single stage bilateral resections and in patients with marginal lung function. As it is technically more demanding in inflammatory conditions than lung cancer it should be attempted by experienced surgeons. The conversion rate of video-assisted thoracoscopic surgery (VATS) to thoracotomy ranges from $3 \%$ to $14 \%$ with majority of the cases due to bleeding and dense adhesions 


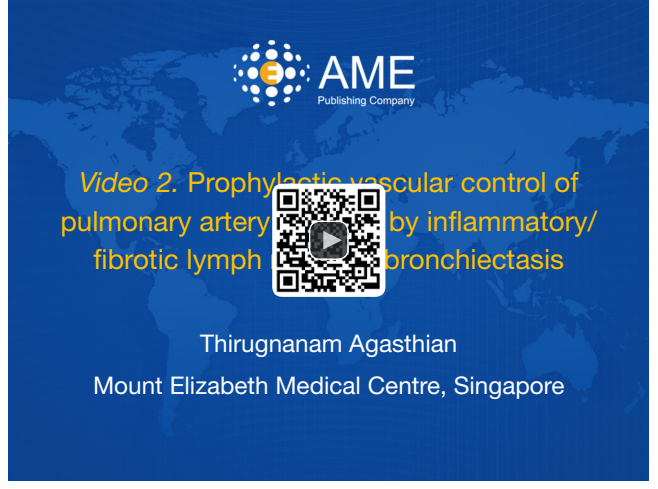

Figure 4 Prophylactic vascular control of pulmonary artery involved by inflammatory/fibrotic lymph nodes in bronchiectasis (19). Available online: http://www.asvide.com/article/view/28193

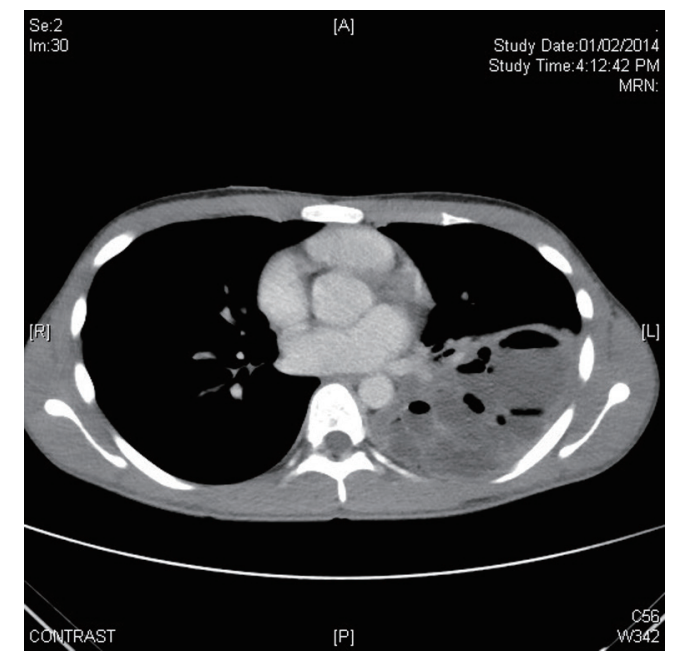

Figure 5 Left lower lobe bronchiectasis.

$(2,13,15-17)$. Sub-xiphoid VATS seems to be a promising alternative to traditional VATS in synchronous bilateral resections due to its ability to do bilateral resections through a single sub-xiphoid incision without repositioning the patient. Though it is limited by its exposure to the posterior and inferior parts of the chest, this can be overcome by the use of additional ancillary ports if necessary.

\section{Types of resections}

In $80 \%$, surgery is done for unilateral disease. Lobectomy $(60 \%)$ is the commonest procedure followed by segmentectomy (25\%) and pneumonectomy (10\%). Lower lobes were the commonest to be resected $(60 \%)$ followed

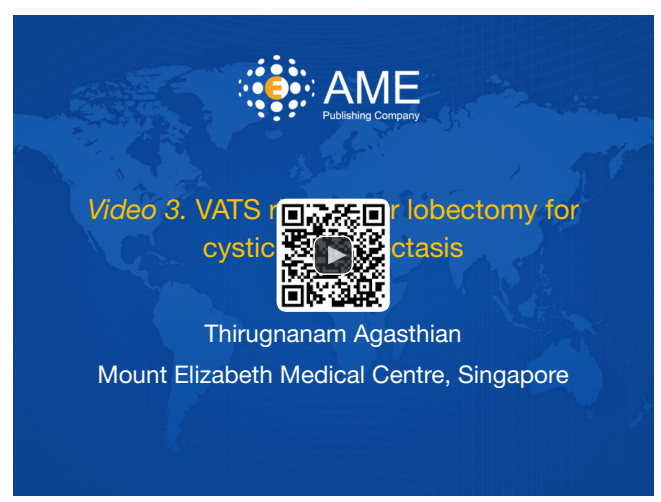

Figure 6 VATS right lower lobectomy for cystic bronchiectasis (20). VATS, video-assisted thoracoscopic surgery.

Available online: http://www.asvide.com/article/view/28195

by lingular and middle lobes. Pneumonectomy is more common where tuberculosis is prevalent (18). Completion pneumonectomy for bronchiectasis is about $6 \%$. Less common resections include bi-lobectomy, lobectomy with segmentectomy and wedge resections. Ten percent are for repeat resections for recurrent progressive disease (12-16).

\section{Surgical tips and pitfalls}

Pleural space problems like prolong air leaks and empyema can be minimised by meticulous lysis of all adhesions with complete mobilisation of all lobes to allow full postoperative re-expansion of the remaining lung. Dense vascular chest-wall and hilar adhesions should when possible be meticulously dissected with sharp dissection using cautery or energy source to minimise blood loss and parenchymal air leaks. Hydrogen peroxide wash or argon beam coagulator can be useful in troublesome chest-wall bleeding. Prophylactic control of hypertrophied bronchial arteries is important. Injury to the pulmonary artery and its branches can be troublesome due to fibrosis and scarring from repeated infections and encasement by hypertrophied calcified lymph nodes. Prophylactic control of main pulmonary artery or intra-pericardial dissection and control of major vessels is useful in such cases $(9,16)$ (Figure 4).

Due to chronic inflammation of the lung and airway, it can be consolidated with entrapped pus and air making surgical manipulation and dissection difficult. Exposure in such cases can be improved by cautery decompression (Figures 5,6). To decrease incidence of air leak fissure-less lobectomy should be done especially when the fissures are not well formed or fused (21). Whenever possible 


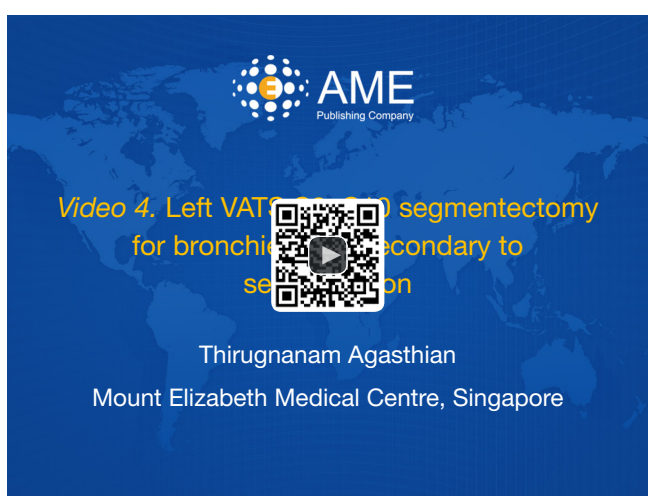

Figure 7 Left VATS S9, S10 segmentectomy for bronchiectasis secondary to sequestration (22). VATS, video-assisted thoracoscopic surgery.

Available online: http://www.asvide.com/article/view/28196

bronchus should be divided early before mobilising the lobe or segment to minimise spillage of secretions to healthy segments of the lung. To decrease risk of BPF devascularisation of the bronchus is minimised by avoiding extensive bronchial and peri-bronchial lymph node dissection (Figures 6,7). The bronchial stump should be divided as short as possible with prophylactic buttressing by vascularised tissue like muscle, pleura, omentum, thymus, pericardium or pericardial fat pad $(9,18)$. In my experience tissue glue has been an effective alternative to bronchial stump vascularised tissue buttressing. There has been no $\mathrm{BPF}$ in more than 30 cases of high-risk bronchial stumps where tissue glue was used (unpublished data) (23). When pneumonectomy is done in the background of pleural sepsis or completely fused pleura an extrapleural, intrapericardial pneumonectomy should be done to prevent contamination of the post pneumonectomy space and early safe ligation of hilar structures $(2,18,23)$.

\section{Clinical outcomes}

Most surgical series quote of $50 \%$ to $80 \%$ cure or asymptomatic, $20 \%$ to $30 \%$ symptom improvement and $5 \%$ to $10 \%$ no improvement or worsening of symptoms after surgery. Single most important factor for good surgical outcomes is complete resection with greater than $90 \%$ improvement of symptoms and $60 \%$ cure rate for complete resections versus $70 \%$ symptom improvement and $20 \%$ cure rate for incomplete resections. Localised disease did better than diffuse disease as complete resections were more amenable in the former. Though cure rates are much lower in diffuse disease there is improvement in symptoms, reductions in hospitalisations and antibiotic usage with overall improved quality of life in more than $70 \%$ of patients especially in those with non-functioning localised cystic bronchiectasis on a background of traction bronchiectasis $(2,8,13-15,24)$.

Surgical mortality in the modern era is low about $1 \%$ to $2 \%$. Mortality is higher $(20-30 \%)$ when performed as an emergency, in the presence of active sepsis and for pneumonectomy (24). Morbidity is about $18 \%$ to $23 \%$ with no significant differences in mortality or morbidity between bilateral or localised disease. Common complications include prolonged air leak, atelectasis, arrhythmias and sputum retention. Major complications like re-exploration for bleeding, empyema and BPF is about $3 \%$ (25).

\section{Conclusions}

Current goals of surgery for bronchiectasis are to cure, improve quality of life and prevent life threatening complications after failed medical therapy. Best surgical outcomes are obtained after complete resections for localised disease. Symptom improvement can be obtained by surgery in selective diffuse disease.

\section{Acknowledgments}

Funding: None.

\section{Footnote}

Provenance and Peer Review: This article was commissioned by the Guest Editor (Kamran Ali) for the series "Asia Thoracoscopic Surgery Education Program (ATEP) Special Issue on Inflammatory Thoracic Diseases" published in Fournal of Visualized Surgery. The article has undergone external peer review.

Conflicts of Interest: The author has completed the ICMJE uniform disclosure form (available at http:// dx.doi.org/10.21037/jovs.2018.10.11). The series "Asia Thoracoscopic Surgery Education Program (ATEP) Special Issue on Inflammatory Thoracic Diseases" was commissioned by the editorial office without any funding or sponsorship. The author has no other conflicts of interest to declare.

Ethical Statement: The author is accountable for all 
aspects of the work in ensuring that questions related to the accuracy or integrity of any part of the work are appropriately investigated and resolved. All procedures performed in studies involving human participants were in accordance with the ethical standards of the institutional and/or national research committee(s) and with the Helsinki Declaration (as revised in 2013). Written informed consent was obtained from the patient for publication of this manuscript and any accompanying images.

Open Access Statement: This is an Open Access article distributed in accordance with the Creative Commons Attribution-NonCommercial-NoDerivs 4.0 International License (CC BY-NC-ND 4.0), which permits the noncommercial replication and distribution of the article with the strict proviso that no changes or edits are made and the original work is properly cited (including links to both the formal publication through the relevant DOI and the license). See: https://creativecommons.org/licenses/by-nc-nd/4.0/.

\section{References}

1. Weycker D, Edelsberg J, Oster G, et al. Prevalence and economic burden of bronchiectasis. Clin Pulm Med 2005:4:205-9

2. Agasthian T. Results of surgery for bronchiectasis and pulmonary abscesses. Thorac Surg Clin 2012;22:333-44.

3. Cobanoglu U, Yalcinkaya I, Er M, et al. Surgery for bronchiectasis: the effect of morphological types to prognosis. Ann Thorac med 2011;6:25-32.

4. Ashour M, Al-Kattan KM, Jain SK, et al. Surgery for unilateral bronchiectasis: results and prognostic factors. Tuber lung Dis 1996;77:168-72.

5. Ashour M. hemodynamic alterations in bronchiectasis. A basis for new classification of the disease. J Thorac Cardiovasc Surg 1996;112:328-34

6. Kutlay H, Cangir AK, Enon S, et al. Surgical treatment in bronchiectasis: analysis of 166 patients. Eur J Cardiothorac Surg 2002;21:634-7.

7. Yu JA, Pomerantz M, Bishop A, et al. Lady Windermere revisited: treatment with thoracoscopic lobectomy/ segmentectomy for right middle lobe and lingular bronchiectasis associated with non-tuberculous mycobacterial disease. Eur J Cardiothorac Surg 2011;40:671-5.

8. Al kattan KM, Essa MA, Hajjar WM, et al. Surgical results for bronchiectasis based on functional and morphological classification. J Thorac Cardiovasc Surg 2005;130:1385-90.
9. Thirugnanam A. Video-assisted thoracoscopic surgery and open chest surgery in infectious lung diseases. J Vis Surg 2017;3:3.

10. Thirugnanam A. Right video-assisted thoracoscopic surgery (VATS) lower lobectomy for bronchiectasis. Asvide 2016;3:514. Available online: http://www.asvide.com/ articles/1289

11. Agasthian T. Selective bronchial blocker of right intermediate bronchus for VATS right lower lobectomy in a bronchiectasis patient with previous left upper lobectomy. Asvide 2018;5:847. Available online: http:// www.asvide.com/article/view/28191

12. Ashour M, Al kattan K, Rafay MA, et al. Current surgical therapy for bronchiectasis. World J Surg 1999;23:1096-104.

13. Agasthian T, Deschamps C, Trastek VF, et al. Surgical management of bronchiectasis. Ann Thorac Surg 1996;62:976-8; discussion 979-80.

14. Zhang P, Jiang G, Ding J, et al. Surgical treatment of bronchiectasis: retrospective analysis of 790 patients. Ann Thorac Surg 2010;90:246-50.

15. George SA, Loeornado HK, Overholt RH. Bilateral pulmonary resection for bronchiectasis: a 40-year experience. Ann Thorac Surg 1979;28:48-53.

16. Mitchell JD, Yu JA, Bishop A, et al. Thoracoscopic lobectomy and segmentectomy for infectious lung disease. Ann Thorac Surg 2012;93:1033-9.

17. Zhang $\mathrm{P}$, Zhang F, Jiang S, et al. Vats for bronchiectasis. Ann Thorac Surg 2011;91:239-43.

18. Thirugnanam A. Video-assisted thoracoscopic surgery (VATS) pneumonectomy for destroyed lung secondary to tuberculosis (TB) stricture. Asvide 2016;3:519. Available online: http://www.asvide.com/articles/1294

19. Agasthian T. Prophylactic vascular control of pulmonary artery involved by inflammatory/fibrotic lymph nodes in bronchiectasis. Asvide 2018;5:848. Available online: http:// www.asvide.com/article/view/28193

20. Agasthian T. VATS right lower lobectomy for cystic bronchiectasis. Asvide 2018;5:849. Available online: http:// www.asvide.com/article/view/28195

21. Thirugnanam A. Right upper lobe fissureless VATS lobectomy for aspergilloma with bronchial buttressing. Asvide 2016;3:515. Available online: http://www.asvide. com/articles/1290

22. Agasthian T. Left VATS S9, S10 segmentectomy for bronchiectasis secondary to sequestration. Asvide 2018;5:850. Available online: http://www.asvide.com/ article/view/28196

23. Thirugnanam A. Completion pneumonectomy. Asvide 
2016;3:518. Available online: http://www.asvide.com/ articles/1293

24. Mazieres J, Murris M, Didier A, et al. Limited operations for severe multi-segmental bilateral bronchiectasis. Ann

doi: 10.21037 /jovs.2018.10.11

Cite this article as: Agasthian T. Surgery for bronchiectasis. J Vis Surg 2018;4:235.
Thorac Surg 2003;75:382-7.

25. Eren S, Esme H, Avci A. Risk factors affecting outcome and morbidity in the surgical management of bronchiectasis. J Thorac Cardiovasc Surg 2007;134:392-8. 